\title{
The Pro-Angiogenic Growth Factor, FGF-2, as a Host-Response Against Invasive Fungal Infections
}

\author{
Sandeep Vellanki, Eun Young Huh, and Soo Chan Lee \\ South Texas Center for Emerging Infectious Diseases, Department of Biology, \\ Objectives: \\ The University of Texas at San Antonio, TX
}

Angioinvasion and tissue injury are hallmarks of infections mediated by Mucor circinelloides (mucormycosis) and Candida albicans (systemic candidiasis). Several studies have shown that pro-angiogenic Fibroblast Growth Factor2 (FGF-2) is essential for tissue repair. Recently, studies have shown that FGF-2 treatment increased survival rates of murine models of aspergillosis. However, the role of FGF-2 and its regulated angiogenesis is understudied in systemic candidiasis and mucormycosis infections. $C$. albicans is a dimorphic fungus that exhibits yeast and hyphae morphologies. Our lab was able to generate an $M$. circinelloides mutant that exhibits yeast morphology in most conditions. We have previously shown that immune cells such as macrophages and lymphoblastoid cell lines overexpress FGF-2 when challenged with either $C$. albicans or $M$. circinelloides hyphae, but not yeast. The main objective of the current study is to determine if the FGF-2 response from a non-immune cell line, Human Umbilical Vein Endothelial Cells (HUVECs) is specific to hyphae form of fungal pathogens. Our second goal is to identify the fungal factors that affect host FGF-2 expression.

\section{Methods:}

HUVEC cells were challenged with either $C$. albicans or $M$. circinelloides yeast or hyphae strains for 24 hours. PBS was used as a mock control. The supernatants were collected to perform FGF-2 ELISA. To determine if live hyphae are essential for FGF-2 expression, we compared FGF-2 expression in HUVECs by challenging them with either live, heat-killed or PFA treated C. albicans or $M$. circinelloides hyphae. To determine if secreted or released factors from fungi are linked with host FGF-2 overexpression, we have grown the fungal pathogens overnight in tissue culture medium, and then we used the spent medium to infect HUVECs.

\section{Results:}

Our results show that HUVECs challenged with $C$. albicans or $M$. circinelloides hyphae show a significant increase in FGF-2 expression when compared to their yeast counterparts. Unlike $M$. circinelloides, only viable $C$. albicans hyphae is linked with increase in FGF-2 response. We also found that $C$. albicans strains deficient in candidalysin cannot increase host FGF-2 expression. Our data also show that secreted factors from $M$. circinelloides hyphae, but not yeast causes an increase in host FGF-2 expression. We also found that cell damage is significantly reduced in monocultures pre-treated with FGF-2 nAb followed by challenge with $M$. circinelloides

\section{Conclusion:}

Host FGF-2 response is specific to hyphae form of fungal pathogens. Our results suggest that secretion of candidalysin by $C$. albicans hyphae into host cells is essential to provoke an FGF-2 response. Hyphae specific factors from $M$. circinelloides can increase host FGF-2 response. Our future studies will focus on determining the role of FGF-2 in murine models of candidiasis and mucormycosis.
Increase in host FGF-2 response is specific to hyphae form of $C$. albicans
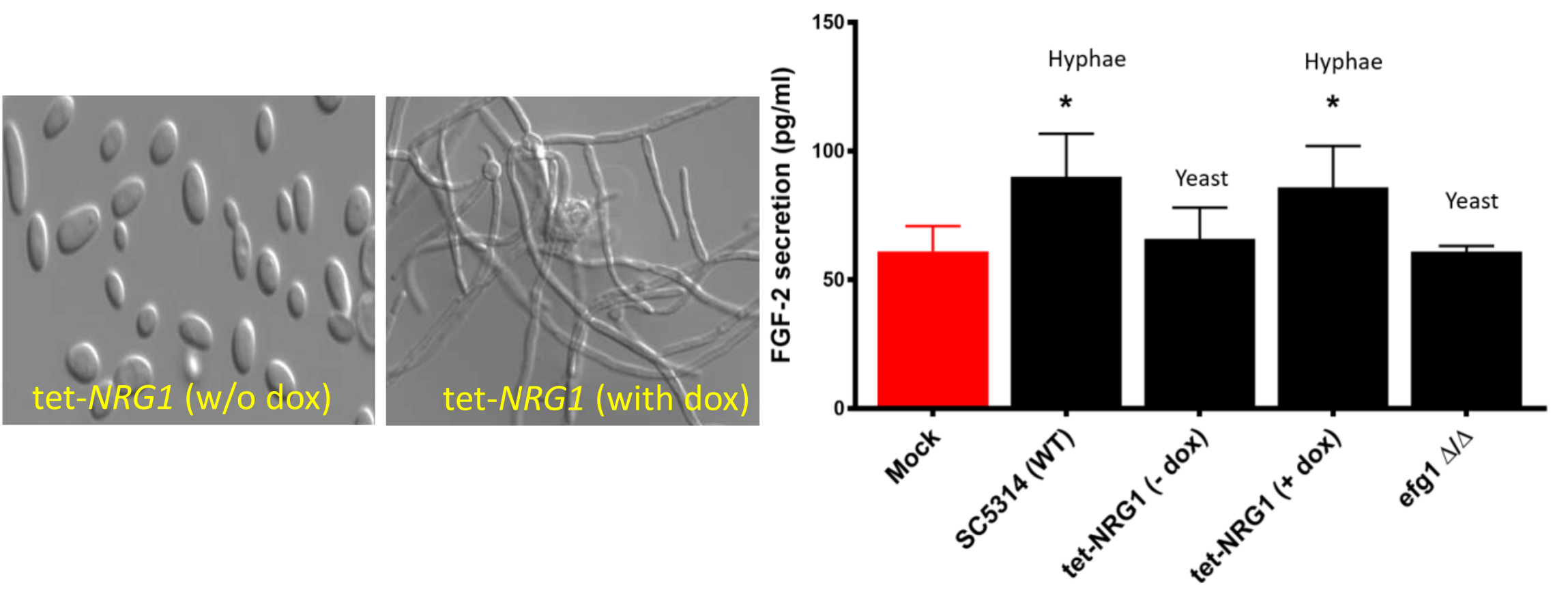

Only viable $C$. albicans hyphae is linked with increase in FGF-2 response

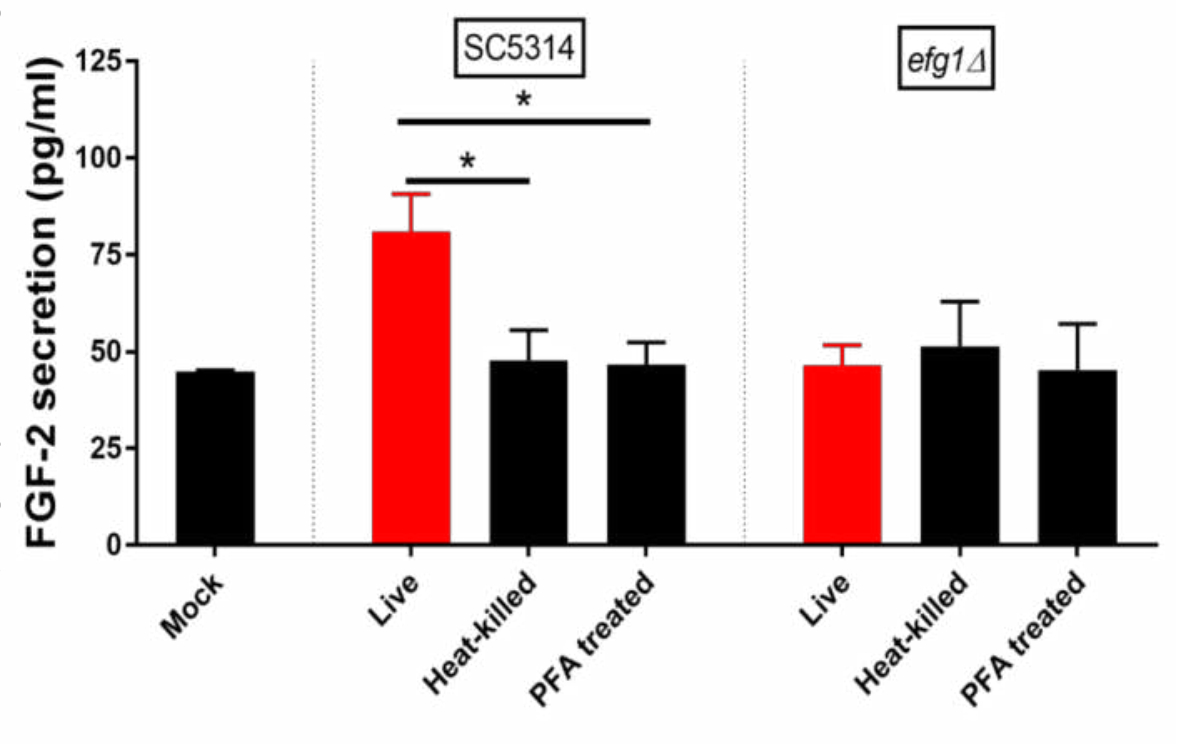

Candidalysin is linked with increase in FGF-2 expression

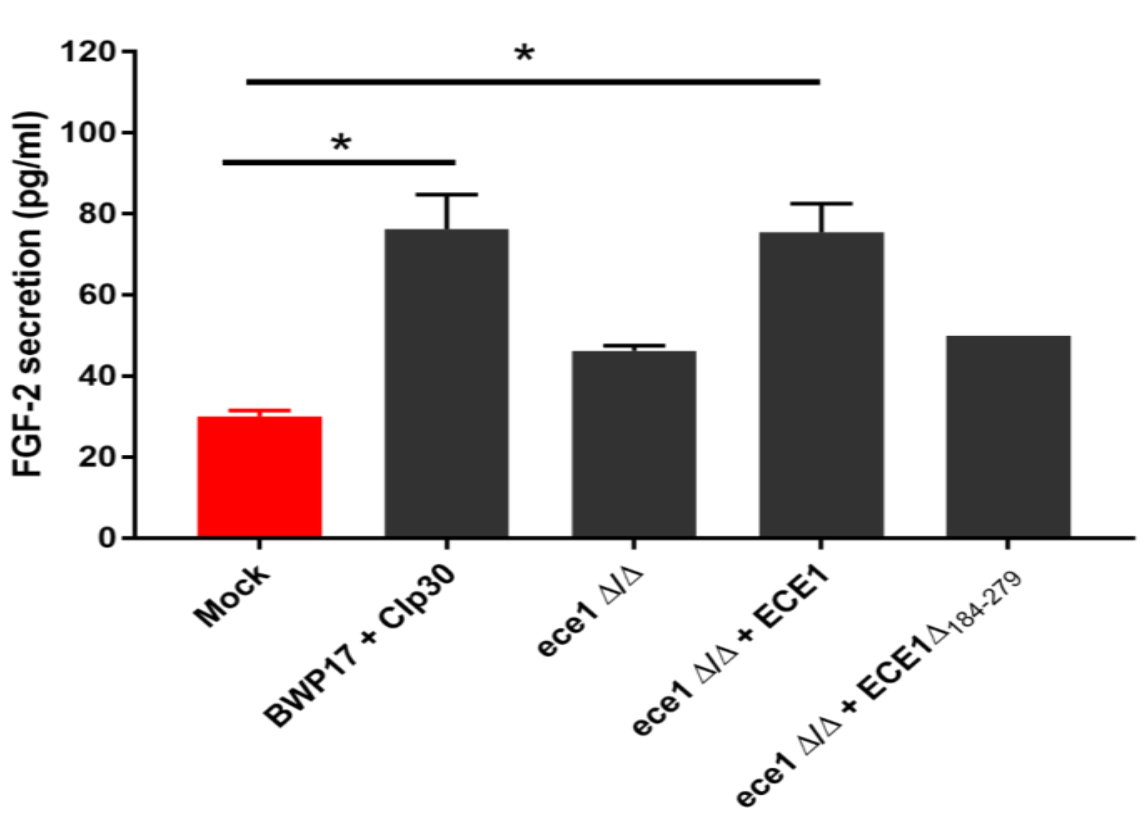

Increase in host FGF-2 response is specific to hyphae form of Mucor
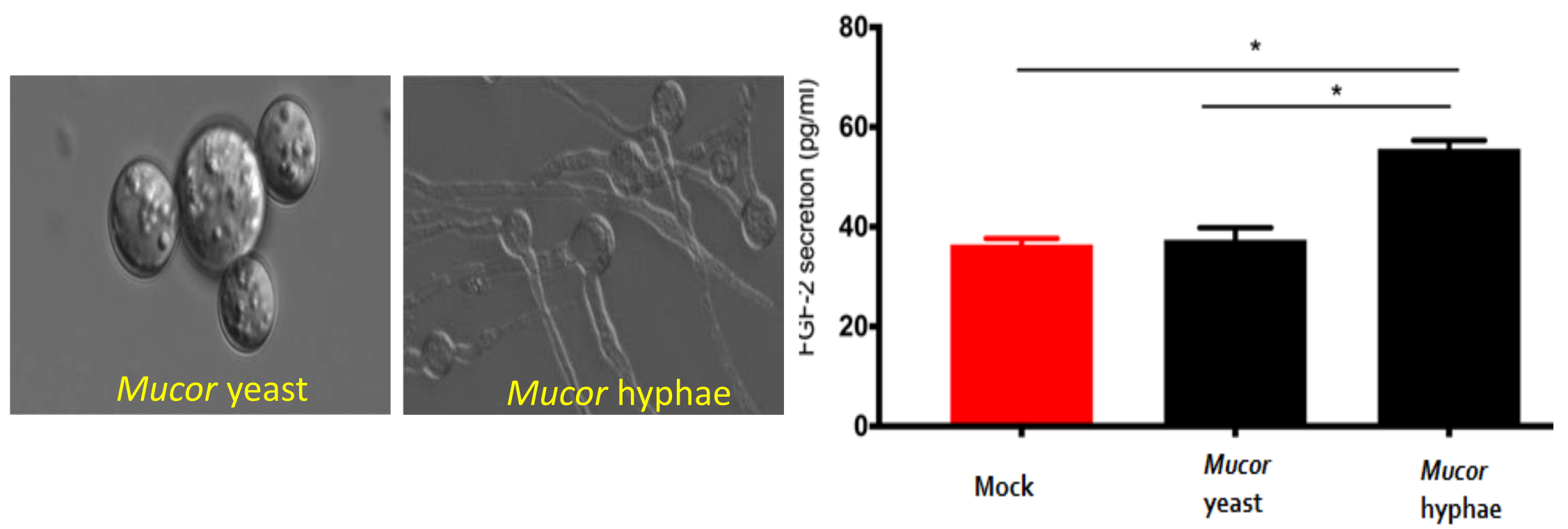

Secreted/released proteins from Mucor is linked with FGF-2 expression

Blocking of FGF-2 resulted in lower cell damage by Mucor
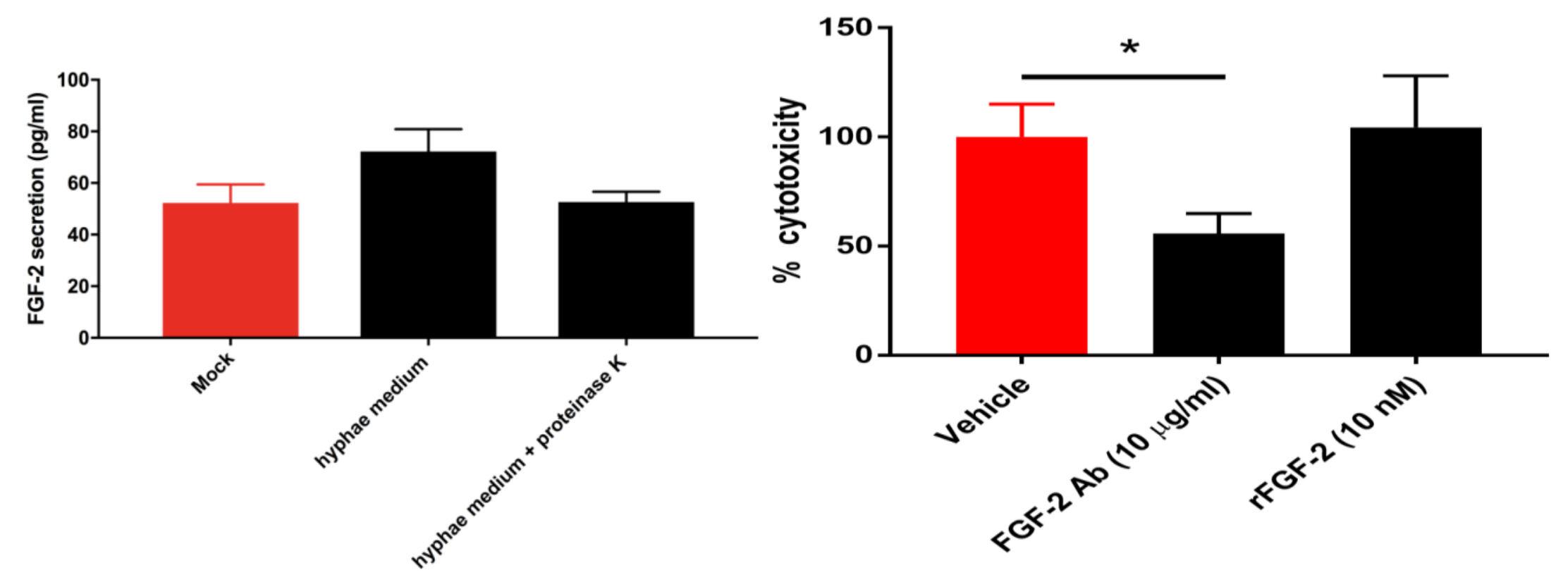